\title{
Integrated Design and Optimization of a Direct Drive Axial Flux Permanent Magnet Generator for a Tidal Turbine
}

\author{
O. Keysan ${ }^{1}$, A. S. McDonald ${ }^{2}$ and M. Mueller ${ }^{3}$ \\ ${ }^{1-3}$ School of Engineering, Institute for Energy Systems \\ University of Edinburgh \\ King's Buildings, Edinburgh EH9 3JL United Kingdom \\ Phone: +44 (0)1316505629 e-mail: o.keysan@ed.ac.uk, markus.mueller@ed.ac.uk
}

\begin{abstract}
The C-GEN is a novel topology of direct drive air-core permanent magnet generator being developed at University of Edinburgh [1]. The topology has many benefits such as; absence of cogging torque, reduced mass and ease of manufacturing. A $20 \mathrm{~kW}$ prototype test rig and $15 \mathrm{~kW}$ machine for a wind turbine has been manufactured and tested previously. Initial sizing studies for wind turbines indicate that the C-GEN concept will be up to $50 \%$ lighter than conventional iron cored PM direct drive generators [1]. In addition to the applications wind turbines, C-GEN technology can also be implemented for marine energy power take-off systems. To investigate that, a feasibility study is being undertaken in collaboration with two wave and two tidal energy companies.
\end{abstract}

In this paper, design and optimization method of an axial flux permanent magnet generator for a tidal energy converter device has been investigated. An analytical optimization tool is designed that combines electromagnetic, structural and thermal aspects of the machine design. A genetic algorithm optimization method has been utilized based on the operation conditions of generator and pre-defined constraints on dimensions and material limitations. The output of the analytical design tool is compared with the electromagnetic FEA simulations. The results showed that proposed analytical calculation method is consistent with FEA results

\section{Key words}

Direct drive, permanent magnet generator, integrated design, tidal energy, genetic algorithm optimization

\section{Introduction}

Marine energy -i.e. wave and tidal energy- converter developers tend to focus their effort on the design and optimization of prime mover and then using off-the-shelf electrical systems for electricity generation. These offthe-shelf components may include a gearbox or hydraulic system to increase the speed of the slow prime mover and a conventional high speed generator. The use of gearboxes and hydraulic pumps introduce a decrease in efficiency and increase in operational and maintenance cost which is a vital factor in off-shore renewable energy converters [2].

Direct drive systems have been successfully demonstrated in the wind industry $[3,4]$. However, as the trend of direct drive systems in marine energy is increasing there is still little expertise and experience in this area. Some distinct devices with direct drive power take-off systems in wave and tidal energy are AWS (Archimedes Wave Swing) [5], Open Hydro [6] and Clean Current [7].

One of the on-going projects that University of Edinburgh takes place is to investigate the feasibility of C-GEN concept for two tidal and two wave energy converters. In this paper, the coupled design and optimization method of a $550 \mathrm{~kW}$ axial flux generator will be investigated. The generator is designed for the Scotrenewables Marine Power Ltd. SRTT tidal energy converter [8].

The prime movers in renewable energies have low rotational speeds. For a tidal current turbine the rotational speed of the blade is limited by $30 \mathrm{rpm}$ due to environmental protection policies [9]. Moreover, the blade designers are forced to reduce the rotational speed in order to keep blade's tip speed ratio in a specific range. Thus, the maximum speed of a tidal turbine is between 20-25 rpm, with a much smaller average due to tide characteristics. In order to provide torque and power requirements at this speed range, the diameter of the generator should be increased. It is showed in [1] that with the increased diameter the structural mass -needed to keep a constant air gap clearance- increases enormously. The mass of the generator directly increases the cost of the generator itself, logistics and installation costs. More over, in a floating power converter device as SRTT, the mass of the generator is limited by the predefined buoyancy of the device. 


\section{Integrated Design}

Conventional design of electrical machines usually begins with electromagnetic design. After designing and optimising the electromagnetically active materials of the generator, the mechanical structure is inserted to the design. Then, the thermal analysis of the machine is performed to verify the operating conditions of the generator and extra cooling paths and forced cooling methods are introduced if necessary. This whole process may include a few iterations which is time consuming and costly. More importantly, since the different aspects of the design are not well coupled, the output usually does not give the optimum solution for specific application.

For the design problem presented in this paper, an integrated design and optimization method has been used that combines electromagnetic, structural and thermal design of the machine.

\section{A. Electromagnetic Design}

Cross-section view of an axial flux C-GEN machine and main dimensions are shown in Figure 1. The generator is an air cored generator so there are no attraction forces between stator and rotor. Lack of stator iron decreases electromagnetic performance but it is not comparable with the added advantages in structural design.

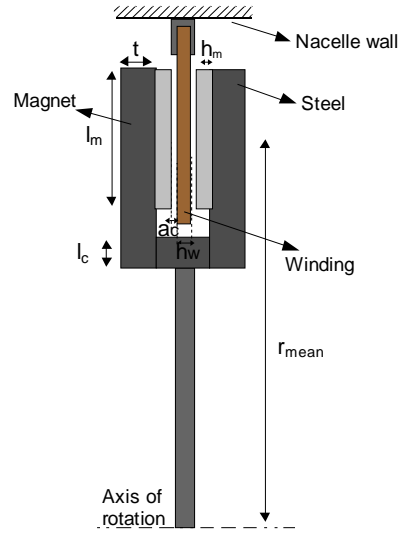

Figure 1 - Half cross section view of axial flux C-GEN machine

Air gap flux density is calculated using a lumped parameter magnetic circuit. The details of the calculations have been presented in $[1,10]$. The proposed lumped parameter method has agreement with FEA results over a wide range. Suppose the air gap flux density is distribution is sinusoidal with a peak value of $\hat{B}$, then the flux distribution can be approximated as follows using the machine dimensions given in Figure 2.

$$
\begin{gathered}
B(\theta)=\hat{B} \cdot \cos \left(\frac{\pi}{\theta_{p}} \theta\right) \quad \text { For } \mathrm{R}_{\mathrm{i}}<\mathrm{r}<\mathrm{R}_{\mathrm{o}} \\
\theta_{p}=\frac{2 \pi}{N_{p}}
\end{gathered}
$$

Then the peak flux linkage can be expressed as;

$$
\hat{\lambda}=\frac{4 \cdot \hat{B} \cdot N_{t} \cdot\left(R_{o}{ }^{2}-R_{i}{ }^{2}\right)}{N_{p}{ }^{2}\left(\theta_{o}-\theta_{i}\right)}\left(\cos \left(\frac{N_{p}}{2} \theta_{i}\right)-\cos \left(\frac{N_{p}}{2} \theta_{o}\right)\right)
$$

Where $\mathrm{N}_{\mathrm{t}}$ is number of turns of coil and $\mathrm{N}_{\mathrm{p}}$ is the number of poles. Again assuming a sinusoidal flux linkage distribution;

$$
\lambda=\hat{\lambda} \cdot \sin (w t)
$$

Then, induced EMF per coil can be obtained by the wellknown Faraday's Law. Then phase currents, terminal voltage and efficiency can be calculated using the phase resistance and inductance. Notice that, the resistances should be updated according to the temperature rise in the windings. This is an iterative process since the increase in coil resistances also increases the coil copper loss.
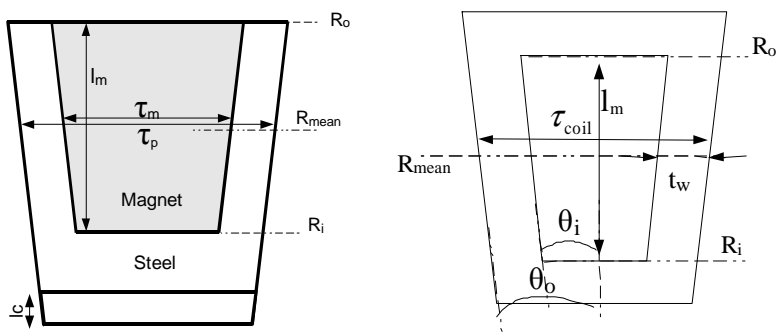

Figure 2 - C-GEN core and coil - front view

\section{B. Structural Design}

In conventional permanent magnet generators, the rotor structure and bearing should be strong enough the keep the air gap clearance against large attraction forces between rotor and stator. The main advantage of C-GEN topology is the absence of these forces due to air-cored structure which allows lighter and cheaper machines to be built. But still, the C-core structure has to withstand the magnetic attraction forces between magnets, reaction torques and gravitational forces. Details of these forces have been presented in [10]. The Maxwell stress between opposing magnets can be defined as;

$$
q=\frac{\hat{B}^{2}}{2 \mu_{0}}
$$

Integrating these stress along radial direction, the $\mathrm{C}$-core can be modelled with a trapezoidal characteristics force applied on a simple beam with one side is fixed and other side is free as given in Figure 3.
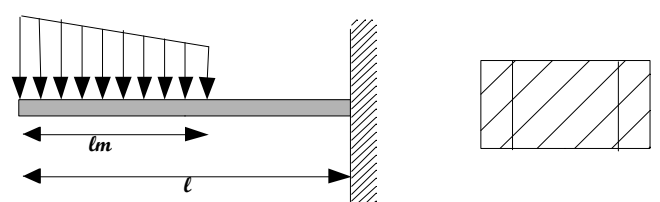

Figure 3 - Beam model and cross section of core

Notice that, the deflection of core is also an iterative process since as the core deflects the distance between magnets decreases which results in increased Maxwell stress and increased deflection.

Besides the deflection in C-core, there will a deflection and torsion in rotor torque arm and shaft due to reaction torques. The torque arm is modelled with a six-arm 
hollow rectangle bars and shaft is modelled with a hollow cylinder as given in Figure 4. The wall thickness of these structures should be adjusted according to required torque and machine radius. There may be another deflection in torque arms when the machine axis is placed vertically i.e. during transportation or maintenance. The deflection at this position should be smaller than the air gap clearance to prevent damage to coils.
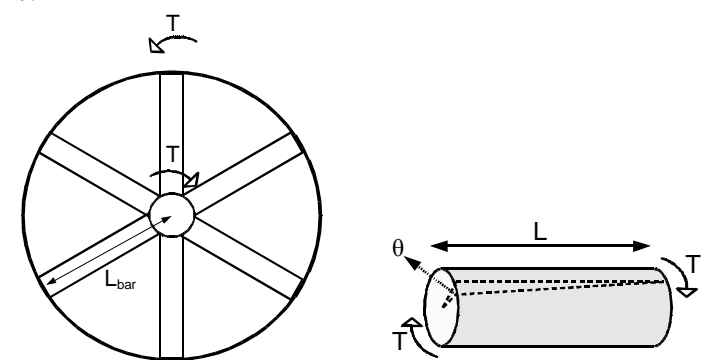

Figure 4 - Torque arm structure and torsion in shaft

\section{Thermal Design}

Thermal design of generators for marine renewable energy converters is quite important because the thermal performance of the generator may be increased due to the low temperatures and better heat transfer ratios in sea conditions. As a result, a higher power can be drawn from a generator or size and the mass of the generator can be reduced, utilizing this thermal advantage [11]. This advantage can be mostly exploited with a flooded machine. On the other side, flooding a generator introduces problems on coating and marinisation of other materials. Thus, flooding is not covered in this study.

The temperature of the windings and the structure is estimated using a lumped-parameter steady state thermal circuit as explained in detail in [12]. Extra heat transfer paths between generator and circulating water across the nacelle have been considered to increase thermal performance. The forced air cooling effect can be modelled in thermal circuit by some convectional thermal resistances.

The heat is directly generated in windings due to copper losses and eddy current losses. Due to low rotational speed and electrical frequency the eddy losses are small compared to copper losses. The approximated winding temperature is then used to calculate the increase in the stator resistance as follows;

$$
R_{t h}=R_{a m b}\left(1+\alpha\left(T_{w}-T_{a m b}\right)\right.
$$

Where $R_{t h}$ is the resistance of the winding in current temperature, $R_{a m b}$ is the resistance at ambient temperature, $\alpha$ is the temperature coefficient for copper, $T_{w}$ is the current winding temperature and $T_{a m b}$ is the ambient temperature.

Also, this calculation is iterative as copper losses increase the temperature of the winding increases resulting in a higher copper loss. After the results are converged, the efficiency, terminal voltage and efficiency of the current design can be calculated.

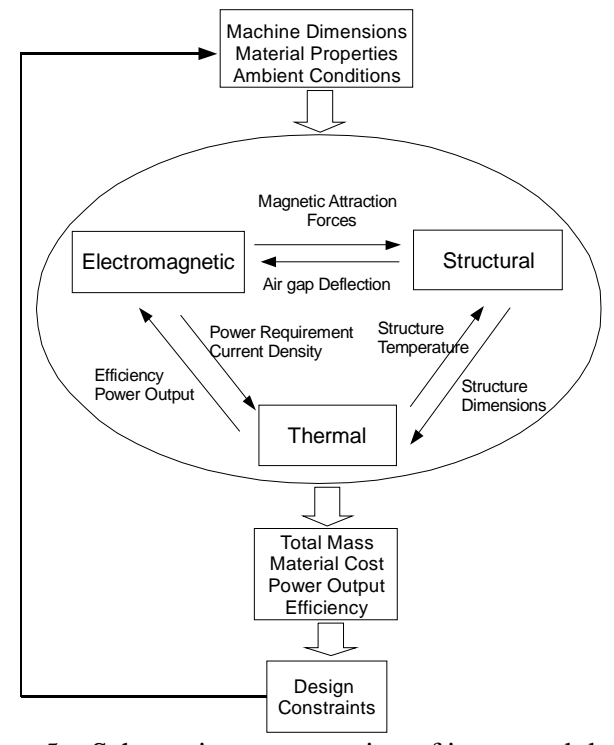

Figure 5 - Schematic representation of integrated design

The schematic of the integrated design is plotted in Figure 5 with the main interactions between different design steps. This kind of approach to machine design minimize the period to final design and has a better chance in finding the optimum solution to the defined design problem.

\section{Optimization Process}

The proposed integrated design is very suitable for an optimization algorithm. The outputs of various designs can be compared according to the desired generator characteristics. The design of an electrical machine process is a non-linear problem with many local minimums and design constraints. The proposed C-GEN topology needs 21 inputs to define generator dimensions. Various analytical optimization techniques can be utilized but genetic algorithm (GA) optimization seems to be the best method for electrical machine design [13]. GA doesn't need any derivative information which is desirable if the search space contains discontinuities. Also, it spans whole search space which minimizes risk to pick a local minima. Another optimization method can also be used but defining the cost function accurately has a higher priority than the method used.

Consider the machine is to be optimized at $n$ discrete steady state operating conditions. Then the cost function can be defined as follows;

$$
F(x)=f_{\text {material }}(x)+\sum_{i=1}^{n}\left(-p_{i} \cdot f_{\text {income }}(x)+w_{i} \sum f_{\text {constraint } s}(x)\right)
$$

$f_{\text {material }}$ (material cost): The material cost does not change with operating conditions. The material cost can be expressed explicitly as follows;

$$
f_{\text {material }}(x)=K .\left(m_{c s} \cdot p_{c s}+m_{c} p_{c}+m_{m} p_{m}+m_{s s} p_{s s}\right)
$$

$\mathrm{m}, \mathrm{p}$ is for mass and price per $\mathrm{kg}$ of materials namely; core steel, copper, magnet and structural steel. $\mathrm{K}$ is a factor that can be used for manufacturing or assembly cost. 
$f_{\text {income }}$ (electricity generation income): The electricity income should be calculated for each operation condition and total value is the multiplication of each value with the probability density of each interval $\left(\mathrm{p}_{\mathrm{i}}\right)$.

$$
f_{\text {income }}(x)=k_{1} \cdot k_{2} \cdot p_{\text {elec }} \cdot P_{\text {out }}
$$

$\mathrm{k}_{1}$ is for availability of the generator - assumed to be $0.95, \mathrm{k}_{2}$ is the efficiency for power electronics and grid connection $-0.93, \mathrm{p}_{\mathrm{elec}}$ is the price of electricity per Watt over ten years - $£ 14.4 / \mathrm{W}, \mathrm{P}_{\text {out }}$ is the output power for that operation condition.

$f_{\text {constraints }}$ (penalty function): In order to force the optimization algorithm through the desired design, penalty functions should be carefully defined. The value of the penalty functions is zero between the defined minima and maxima, otherwise it is a second order parabolic function.

$$
\begin{array}{ll}
f(x)=0 & \mathrm{x}_{\min }<\mathrm{x}<\mathrm{x}_{\max } \\
f(x)=k \cdot\left(x-x_{\max }\right)^{2} & \mathrm{x}>\mathrm{x}_{\max } \\
f(x)=k \cdot\left(x-x_{\min }\right)^{2} & \mathrm{x}<\mathrm{x}_{\min }
\end{array}
$$

where $\mathrm{k}$ is the weight constant for each constraint function. The following constraints are defined in the optimization process;

- Power output interval

- Temperature

$$
\begin{array}{ll}
\circ & \text { Winding } \\
\circ & \text { Magnet }
\end{array}
$$

- Deflections

$$
\begin{array}{ll}
\circ & \text { Core deflection as a ratio of air gap } \\
\circ & \text { Torque arm } \\
\circ & \text { Shaft }
\end{array}
$$

- Flux density of core

- Maximum phase voltage

Notice that, there are no constraints for mass and efficiency since these are explicitly included in material cost and electricity income functions. By this way, the optimization program does not give the lightest or most efficient generator but it gives the most optimum design for a wide range of operation conditions.

Generic algorithm is performed with an initial population of 1500 , and the optimization is performed for 40 generations with a population of 250 . The minimum and mean cost for each generation step is given in Figure 6. This graph shows the optimization converges to a solution.

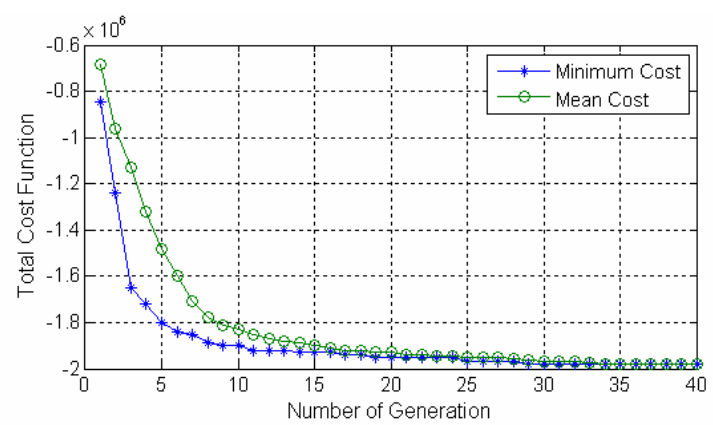

Figure 6 - Minimum and mean cost for each generation

\section{Optimized Generator}

As mentioned earlier, the generator optimization is prepared for the SRTT which is presented in Figure 7. The SRTT is a floating tidal turbine with two vertical blades. SRTT has significant advantages in installation and maintenance costs [9].

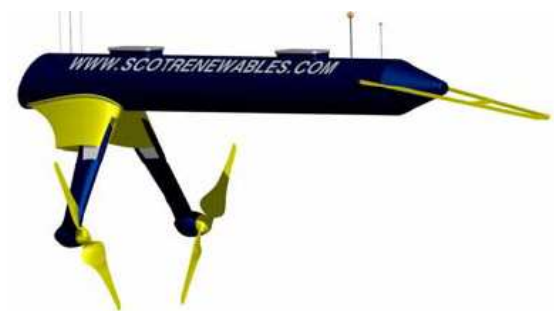

Figure 7 - SRTT - Scotrenewables Tidal Turbine - by courtesy of Scotrenewables

The optimization algorithm has been performed using ten discrete operation conditions of SRTT, with dimension and mass constraints of the device. Ambient temperature is assumed to be $10{ }^{\circ} \mathrm{C}$. The main data for the generator is as follows;

Table 1 - Main data for optimised generator

\begin{tabular}{|l|l|}
\hline Power Rating & $550 \mathrm{~kW}$ \\
\hline Outer Diameter & $3 \mathrm{~m}$ \\
\hline Axial Length & $0.7 \mathrm{~m}$ \\
\hline Weight & 13 tonnes \\
\hline Peak Efficiency & $95.6 \%$ \\
\hline Weighted Average Efficiency & $88.8 \%$ \\
\hline
\end{tabular}

The efficiency data for different rotational speeds have been presented in Figure 8. Although the rated power of the generator is $550 \mathrm{~kW}$, it can generate up to $750 \mathrm{~kW}$ at $20 \mathrm{rpm}$ with a decreased efficiency.

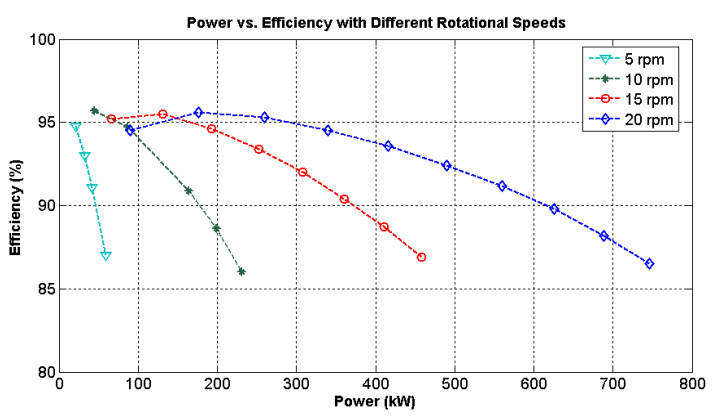

Figure 8 - Efficiency of the optimized design

In Figure 9, the designed generator is shown mounted on the SRTT nacelle with a human figure for scaling. Also, the cut through section of the generator has been presented. The generator is composed of six axially stacked generators. Main advantage of this configuration is decrease in the active and structural material mass. Also the generators can be controlled independently which creates a redundancy. The generator may still operate partially even when there is a problem in some of the stacked generators which is a highly desired feature for offshore power take-off systems. 


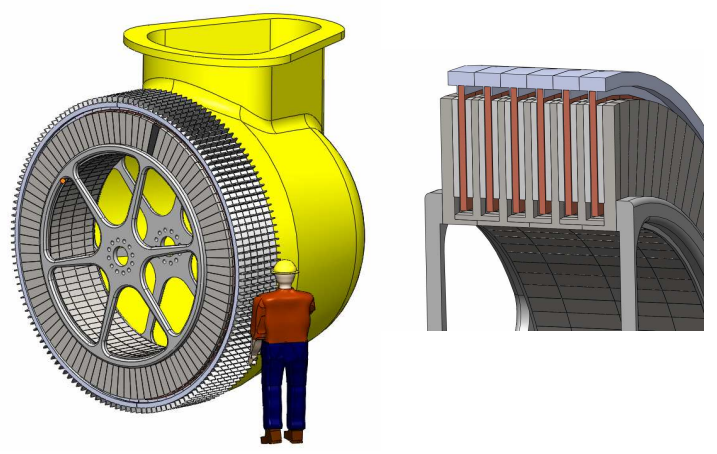

Figure 9 - Generator mounted on SRTT nacelle - Cut through view of generator

\section{FEA Verification}

In order to validate the analytical model, some simulations have been made using finite element analysis (FEA) method. For electromagnetic analysis a half symmetry model is used as shown in Figure 10. The selected magnet type has a remanence flux density of $1.32 \mathrm{~T}$. And the steel has the magnetic characteristics of mild-cast steel. Half cross-section of a single coil is also included in the model for induced voltage and torque calculations. The FEA model shows the part of the core between neighbouring magnets is highly saturated. The maximum air gap flux density is found to be $0.63 \mathrm{~T}$.

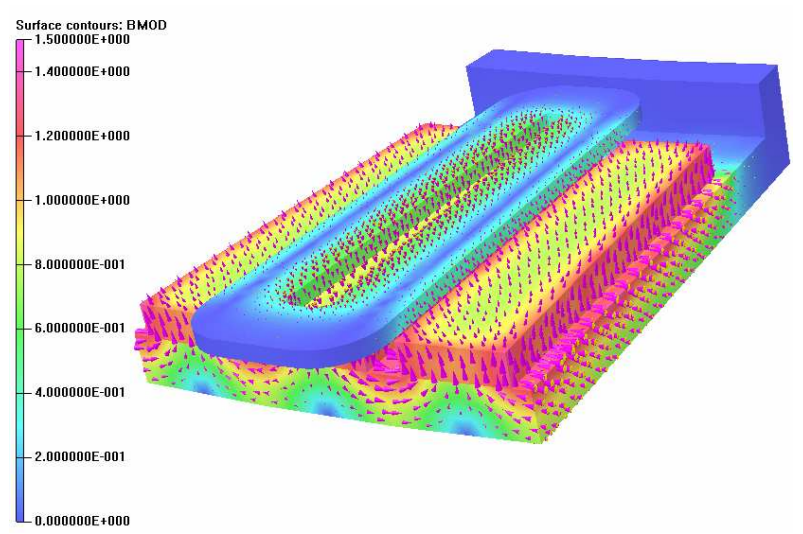

Figure 10 - Electromagnetic FEA simulation- half symmetry

Experiments are performed to validate the analytical thermal model and measure the thermal performance of the air-cored stator but the results are not included in this study.

For structural simulations it is assumed that Young's modulus of steel is $200 \mathrm{GPa}$ and shear modulus is 79.3GPa. Pressure is applied on both sides of the C-core limbs as given in equation 5 . The magnetic attraction force increases as the limbs deflect, so three iterations are performed until the deflection and magnetic attraction force converges. The exaggerated view of deflection in a group of four C-cores is presented in Figure 11.

Maximum deflection using FEA is found to be $0.255 \mathrm{~mm}$ where the analytical model gives $0.273 \mathrm{~mm}$. Both the analytical and FEA results are smaller than design constraint $-10 \%$ of the air gap clearance.

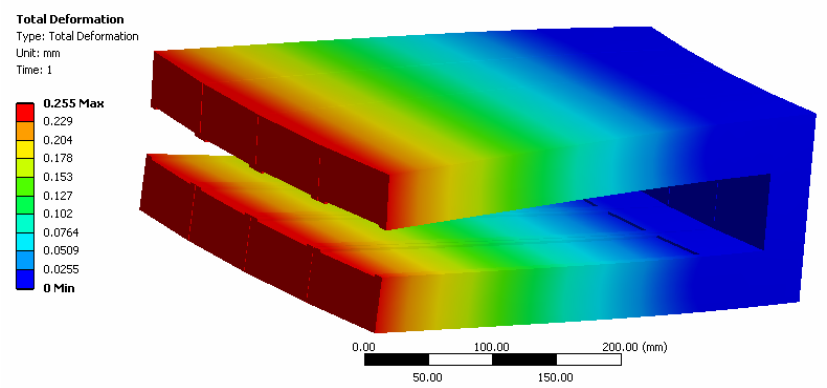

Figure 11 - Structural FEA simulation -exaggerated view

The analytical model and FEA results are compared in Table 2. The table shows both electromagnetic and structural results are well agreed with the FEA results. Largest error is in structural modelling. But the deflection in analytical model is bigger which can be assumed as a safety margin to manufacturing tolerances.

Table 2 - Comparison of analytical model and FEA

\begin{tabular}{|l|c|c|c|}
\hline & $\begin{array}{c}\text { Analytical } \\
\text { Model }\end{array}$ & FEA & Error (\%) \\
\hline $\begin{array}{l}\text { Max. Air Gap Flux } \\
\text { Density }\end{array}$ & $0.65 \mathrm{~T}$ & $0.63 \mathrm{~T}$ & 3.1 \\
\hline $\begin{array}{l}\text { Induced Voltage (at } \\
\text { rated speed, per coil) }\end{array}$ & $850 \mathrm{~V}_{\mathrm{rms}}$ & $829 \mathrm{~V}_{\mathrm{rms}}$ & 2.5 \\
\hline Mechanical Torque & $386 \mathrm{kNm}$ & $372 \mathrm{kNm}$ & 3.7 \\
\hline Maximum deflection & $0.273 \mathrm{~mm}$ & $0.255 \mathrm{~mm}$ & 7 \\
\hline
\end{tabular}

\section{Conclusion}

In this paper, it is shown C-GEN direct drive generator topology is feasible for tidal turbine devices. Integrated electromagnetic, structural, thermal design of the generator enables accurate evaluation of the design. By the genetic algorithm optimization it is easier to obtain the most suitable design for the current application over all operating conditions. This process is very important in variable speed power take-off systems to obtain the most optimum solution. The cost function in the optimization has been modified to reflect more realistic features of manufacturing, material costs and electricity generation income. Also, the well-agreement between analytical model results and FEA simulation results has validated integrated analytical design tool.

The output of the optimization showed that a $550 \mathrm{~kW}$ generator can be designed with a mass of 13 tonnes which is comparable to current hydraulic or gearbox systems. Also, removing these intermediate steps increases the average efficiency almost $90 \%$. Besides the increased efficiency, the removal of hydraulic pumps or gearboxes increases the robustness of the system and decrease maintenance costs. With these features, direct drive C-GEN generator is a promising technology for marine energy applications. 


\section{Acknowledgement}

The work included in this paper was funded by Npower juice. We also acknowledge the support from Scotrenewables Ltd. which is one of the partners in the project.

\section{References}

[1] A.S. McDonald, "Structural analysis of low speed, high torque electrical generators for direct drive renewable energy converters," Ph.D. dissertation, School of Engineering \& Electronics, University of Edinburgh, to be submitted, 2008.

[2] "Garrad Hassan - Phase 2 Market Review, Lightweight Direct Drive Generator," 2006.

[3] D. Bang, H. Polinder, G. Shrestha, and J. Ferreira, "Review of Generator Systems for Direct-Drive Wind Turbines," European Wind Energy Conference \& Exhibition, Belgium, 2008, pp. 1-11.

[4] Y. Duan and R.G. Harley, "Present and future trends in wind turbine generator designs," 2009 IEEE Power Electronics and Machines in Wind Applications, 2009, pp. 1-6.

[5] "AWS - Archimedes Wave Swing,"

http://www.awsocean.com, 2009.

[6] "Open Hydro Technology," http://www.openhydro.com/technology.html, 2009.

[7] "Clean Current," http://www.cleancurrent.com/technology/index.htm, 2009.

[8] "Scotrenewables - SRTT," http://www.scotrenewables.com, 2009.

[9] M. Francis and M. Hamilton, "SRTT Floating Tidal Turbine Production Design Study with Independent Verification," Carbon, 2007.

[10] A. McDonald, M. Mueller, H. Polinder, U.

Edinburgh, and N. Delft, "Structural mass in directdrive permanent magnet electrical generators," IET Renewable Power Generation, EWEC 2007 Special Issue, vol. 2, 2008, pp. 3 - 15.

[11] N. Hodgins, M. Mueller, W. Tease, and D. Staton, "Measurement and modelling of induction generator performance in an oscillating water column wave energy converter," 4th IET International Conference on Power Electronics, Machines and Drives (PEMD 2008), 2008, pp. 76-80.

[12] A. McDonald, I. Portugal, M. Mueller, and J. Shek, "A time series approach to design of a permanent magnet synchronous generator for a direct-drive wind turbine," 2008 18th International Conference on Electrical Machines, 2008, pp. 1-6.

[13] N. Bianchi and S. Bolognani, "Design optimisation of electric motors by genetic algorithms," Electric Power Applications, IEE Proceedings -, vol. 145, 1998, pp. $475-483$. 